\title{
DECISION SUPPORT FOR DISTRIBUTION AUTOMATION: DATA ANALYTICS FOR AUTOMATED FAULT DIAGNOSIS AND PROGNOSIS
}

\author{
Xiaoyu WANG \\ University of Strathclyde - UK \\ xiaoyu.wang@strath.ac.uk
}

\author{
Stephen MCARTHUR \\ University of Strathclyde - UK \\ s.mcarthur@strath.ac.uk \\ Bruce PAISLEY \\ SP Energy Networks - UK \\ bruce.paisley@spenergynetworks.co.uk
}

\author{
Scott STRACHAN \\ University of Strathclyde - UK \\ scott.strachan@strath.ac.uk
}

\begin{abstract}
Distribution Automation (DA) is deployed to reduce outage times, isolate the faulted area, and rapidly restore customer supplies following network faults. Recent developments in Supervisory Control and Data Acquisition (SCADA) and intelligent DA equipment have sought to improve reliability and security of supply. The introduction of such 'intelligent' technologies on distribution networks, where investment in dedicated condition monitoring equipment remains difficult to justify, presents an opportunity to capture constant streams of operational data which can offer a useful insight into underlying circuit conditions if utilised and managed appropriately.
\end{abstract}

The primary function of the NOJA Pole-Mounted AutoRecloser (PMAR) is to isolate distribution circuits from detected faults, while attempting to minimise outages due to transient faults. However, in this process the PMAR also captures current and voltage measurements that can be analysed to inform any subsequent fault diagnosis, and potentially detect the early onset of circuit degradation, and monitor and predict its progression.

This paper details the design and development of an automated decision support system for fault diagnosis and prognosis, which can detect and diagnose evolving faults by analysing PMAR data and corresponding SCADA alarm data. A knowledge based system has been developed, utilising data science and data mining techniques, to implement diagnostic and prognostic algorithms which automate the existing manual process of post fault diagnosis and anticipation, and circuit condition assessment.

\section{INTRODUCTION}

There is a significant drive in the UK to focus on decreasing Customer Minutes Lost (CML) and Customer Interruptions (CIs) [1], and improve customer service. Distribution Automation (DA) has a major role in smart grid implementation, with the primary objectives of improving the security of supply and reliability of distribution power networks.
This research has developed a knowledge-based decision support system (KB-DSS) capable of diagnosing equipment faults associated with PMARs and which is also capable of detecting evolving fault conditions on distribution circuits protected by these PMAR devices. As such the developed system offers decision support to network operators that can provide early notification of incipient circuit faults allowing evasive action to be carried out (reducing CMLs and CIs), and also provide fault diagnosis for targeted circuit maintenance The development of this DSS will be explained in detail and case studies will be used to illustrate its practical application.

\section{CIRCUIT PERFORMANCE AND MANUAL PROCESS}

SP Energy Networks (SPEN) use microprocessorcontrolled Overhead Line (OHL) Pole-Mounted AutoReclosers (PMAR) [3] to ensure a reliable and serviceable supply condition by avoiding unnecessary circuit outages resulting from transient OHL faults [4]. These PMARs represent an example of intelligent DA devices which can automatically isolate the affected area when a fault occurs, expediting the process of fault location and supply restoration while simultaneously recording circuit data which can be used to assess circuit condition [5]. Fig. 1 shows the operation of the PMAR device when confronted with a transient (fleeting) circuit fault and a permanent fault.
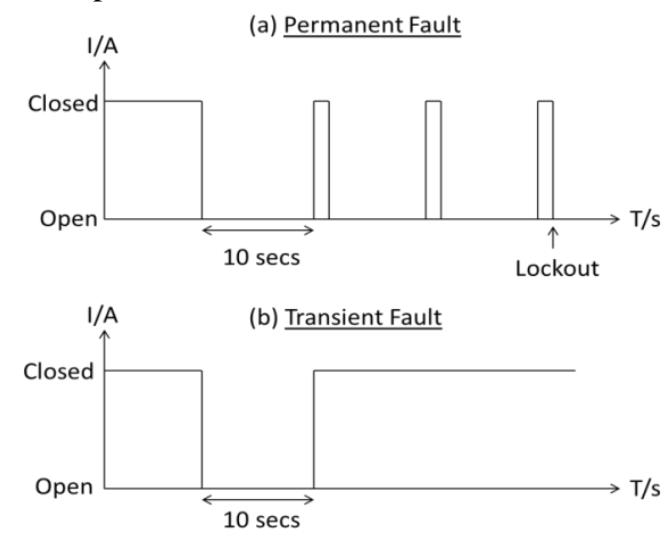

Fig. 1 PMAR operation 
When a fault occurs, the PMAR provides a set number of reclosure attempts (typically 3 attempts, which is set by the DNO). If a reclosure attempt is unsuccessful (i.e. the fault persists), the PMAR will trip almost instantaneously following its reclosure and remain open for a period of 10 seconds before attempting a further reclosure. Following three reclosures, if the fault still persists on the circuit, the PMAR will 'lockout', which suggests that a 'permanent fault' may exist (see Fig. 1 (a) above). However, following a 'lockout', the PMAR can only be reclosed manually via telecontrol by the control engineers.

If the fault has been removed following a PMAR autoreclosure attempt, before reaching 'lockout', this suggests the fault has been a short-lived fault referred to as a 'transient fault'. Fig. 1 (a) illustrates how a transient fault is cleared following the first reclosure attempt of a PMAR.

Furthermore, there is another class of evolving faults arising from the degradation of OHLs which can lead to frequent short-term supply interruptions (i.e. resulting in nuisance tripping), which are defined as 'semi-permanent faults' (SPF). An example of a SPF would be a fault resulting from cracked insulator on a wood pole (which when dry presents no fault condition, but when wet can result in a short circuit fault).

SPFs result in unsolicited PMAR trip activity, referred to as 'Unsolicited Openings' (UO) by SPEN. This classification of PMAR operation represents PMAR trip operations resulting from undiagnosed faults (be they transient or permanent). At present, in order to improve customer service and avoid regulatory penalties, SPEN engineers frequently (and manually) review UOs that occur on the network, in an attempt to ascertain the degree to which the network is affected by this activity. This requires alignment and analysis of a number of data sets, stored in different repositories:

- The Morning Report is the daily report generated manually by the control room team, including the relevant UO and Normally Open Point (NOP) operation activity (e.g. specific PMAR's name, circuit code, etc.) captured from SCADA system information.

- PSALERTS is a data set that archives SCADA alarms which include tripping information (e.g. test trips, UOs, etc.) associated with the particular circuit details (e.g. activity log time, specific PMAR's name, circuit code, etc.).

- PROSPER is a database that includes causal information related to repaired faults, which are generated manually by the maintenance staff. This information contains the causes of faults, the fault clearing time, details of affected equipment.

Fig. 2 shows the manual process currently undertaken, including the three stages of Morning report check, PSALERTS check and PROSPER check.



Fig. 2 Manual process

As illustrated in the diagram, the engineers' focus has been on identifying the problematic PMAR through checking the frequent UOs of the relative circuits. By diagnosing the causes of faults (from checking the fault record in PROSPER) related to the UOs, the analysis may provide a picture of circuit behaviour and underlying conditions. The focus of the UO analysis performed (manually) by engineers has been on identifying problematic circuits and PMAR devices without necessarily focusing on diagnosing the root causes of PMAR operations.

For the purpose of diagnosing root cause problems and predicting evolving faults, this paper describes the design and implementation of a Decision Support System (DSS) that the engineers use, following their manual analysis. The DSS automates the existing approach to analysing and quantifying UO activity associated with PMARs and circuits, while integrating fault diagnostics and also a predictive capability which would alert engineers to incipient fault conditions. This diagnostic and predictive capability is presented as a knowledge based decision support system which mainly makes use of the data captured in the PMAR log files [6], which contains the details of recorded 'pick-up' activities. These include anomaly activities (which do not lead to a trip operation) and fault events (which lead to a trip or lockout operation of the specific PMAR), i.e. the current amplitude of pickup activity, the affected phases, and the time stamp of occurrence and clearance of pick-up activity, etc.

The design of DSS and implementation of the deployed knowledge based system (KBS) will be detailed in the following sections.

\section{DECISION SUPPORT SYSTEM DESIGN}

The key functionality of the decision support system described in this paper is to diagnose PMAR device faults and detect semi-permanent faults responsible for UOs. It will then provide an 'early warning' of future PMAR operation in order to reduce the impact on customers 
before they escalate into more serious and damaging supply outages. This requires analysis of SCADA alarm data and recorded fault details from the PMARs.

Generally, the decision support system provides automated data analysis and prediction, and builds this from visualisation tools developed as part of the research. These visualisation tools also offer the end user advanced functionality when the system is implemented. Fig. 3 shows an activity diagram describing the functionalities in the DSS.

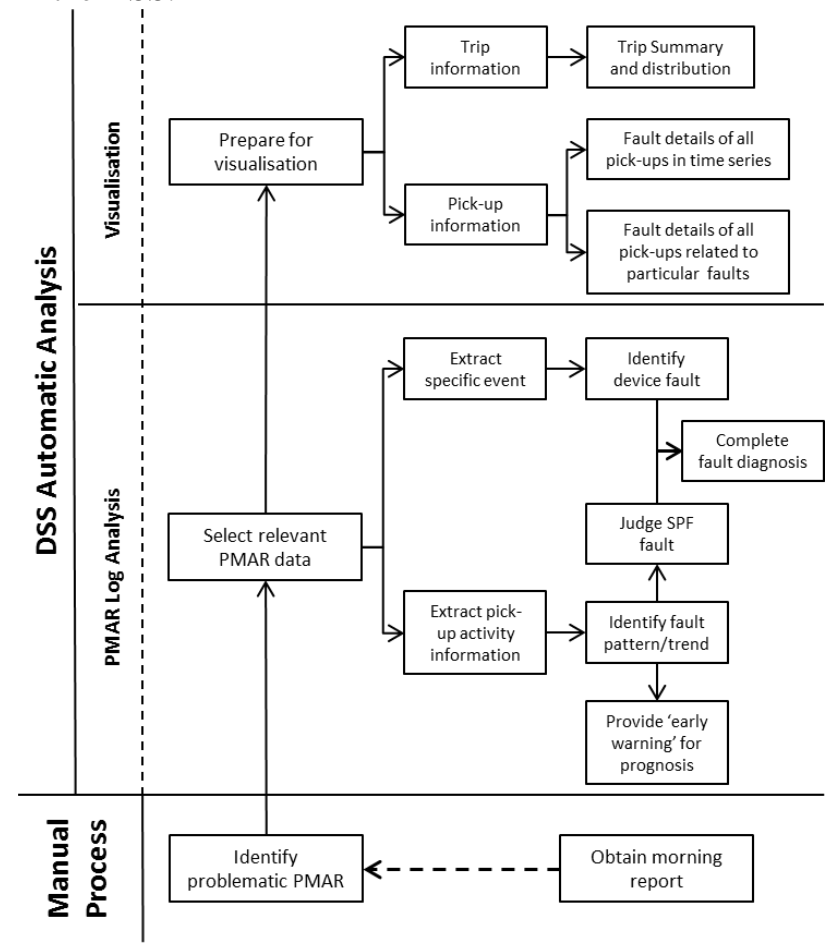

Fig. 3 DSS Activity Diagram

\section{Circuit pick-up activity visualisation analysis}

The visualisation focuses on analysing the details of PMARs' log data after they have been imported into the system. It will automatically derive useful information and allow users to visualise it. This information includes the trips' summary, and pick-up distributions and details for selected time periods independently, which do not necessarily invoke operation of the PMAR but offers an insight into the underlying condition of the circuit. Any specific details (i.e. the amplitudes of fault currents and voltage, fault type, fault duration, etc.) of pick-up activities can be searched for. This facility offers engineers the opportunity to view the trends in underlying circuit pick-up activity leading to PMAR operation. This also assists engineers' decision making for fault diagnosis.

\section{Circuit pick-up activity (PMAR log) analysis}

The PMAR log file analysis is conducted in parallel to the visualisation in the automatic analysis. It is divided into the diagnosis of PMAR device fault, detection of the semi-permanent fault diagnosis and 'early warning' or prediction of degrading PMAR or circuit conditions. The implementations of these functionalities are detailed in the next section of the paper.

As demonstrated in the activity diagram, the DSS will automatically identify the PMAR device faults based on detecting specific warnings or messages related to different categories of device faults, which are stored in the PMAR log file. In order to determine the existence of the SPF, the extracted pick-up information will be evaluated by identifying the patterns and trends. This pick-up data analysis is performed using the KBS whic is based on the experts' knowledge and fault clustering techniques. This can be visualised through the tools developed during this research. Finally, the prognostic functions integrated within the DSS will predict PMAR operations to mitigate the customer interruptions (which may be raised from SPFs) by invoking the prognostic rule developed in the KBS.

\section{AUTOMATED KBS IMPLEMENTATION}

This section outlines the design and different applications of the KBS, including diagnosis of PMAR device fault, detection of semi-permanent faults on the circuits and prognosis of PMAR operations. The functions described are all implemented through a rule-base within the KBS.

\section{Diagnosis of PMAR device faults}

The Main Processor Module (MPM) of the PMAR is responsible for condition monitoring and recording the on-line activity details, which contain information on existing or potential device faults or circuit faults.

Therefore, after the raw log data is imported, the KBS automatically captures and processes it and applies diagnostic rules. Fig. 4 shows how the KBS automatically characterises the following known PMAR faults.

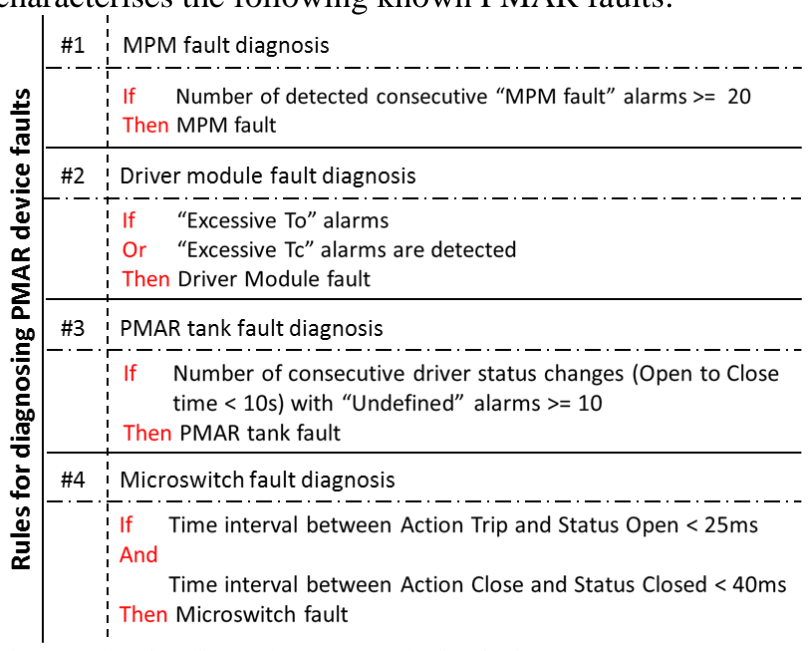

Fig. 4 Rules for diagnosing PMAR device fault

This is the first stage of automated decision support, which focuses on PMAR device fault diagnostics. The next stage of the system concentrates on overhead line fault diagnoses. 


\section{Semi-permanent fault detection on circuits}

The first step of SPF detection is to classify 'pick-up' data into different classifications of PMAR operation and filter the activity caused by potential SPF candidates. Trends of classified SPF candidates can then be used to confirm the existence of SPFs. To assist in the detection of SPFs, another set of rules were developed to classify the PMAR operations through engineers' expert knowledge. The integrated visualisation tool in the DSS allows engineers to observe trends and patterns associated with populations of PMAR operations.

\section{Classification of PMAR operations}

Depending on the operation of PMARs under different fault conditions, the KBS groups 'pick-up' activity into different classifications of PMAR operation (i.e. fault 'pick-up' activity leads to no trip, single trip, multiple trips and lockout). The rules are shown in Fig. 5 classifying the operations in order of increasing severity.



Fig. 5 Rules for classifying PMAR operations

Using these rules the DSS can then identify, map out and prioritise potential SPF activity on circuits which have experienced pick-up activity exceeding acceptable time limits. Then, the network experts can drill into the data for a deeper analysis to detect the trends of pick-up data and identify the existence of SPFs by using the visualisation tool integrated with the DSS.

\section{Trends' analysis for SPF detection}

Following the classifications of PMAR operation, the trends' analysis through the visualisation tool can provide an insight into potential SPFs.

In order to detect the existence of SPFs, four statistical features and trends are defined, analysed and visualised to assist control engineers to make decisions before the SPF trends reach levels that may culminate in a permanent fault resulting in an extended unplanned outage. They are cumulative frequency distribution (CFD) and frequency distribution (FD) of PMAR operations, duration time (DT) of each associated 'pick-up' activity and the interval time (IT) between consecutive pick-ups. An increasing rate in the CFD suggests the faults becoming more frequent. If the corresponding FD exceeds the average number of PMAR operations then this indicates an evolving fault on the circuit. For the purpose of confirming the existence of SPFs, where an increase in DT and a decrease in IT occurs then this suggests the circuit's condition is worsening (i.e. pickups are lasting longer and occurring more frequently) and can be used to categorise the stage of maturity of the SPFs.

\section{Fault prognosis resulting in PMAR operations}

In addition to its fault diagnosis functionality, this system offers a prognostic capability capable of predicting or anticipating potential PMAR operation.

To achieve the prognostic function, the system was developed using a structured method of data mining to derive predictive rules that were designed to operate on anomalous 'pick-up' activity, yet led to a PMAR operation (previously classified as "FP" in Fig. 5).

The application of a K-Means algorithm enables the segmentation and clustering of the features of FP selected for indicating PMAR operations. Fig. 6 shows the predictive rule after K-Means clustering based on the actual network data has been undertaken.

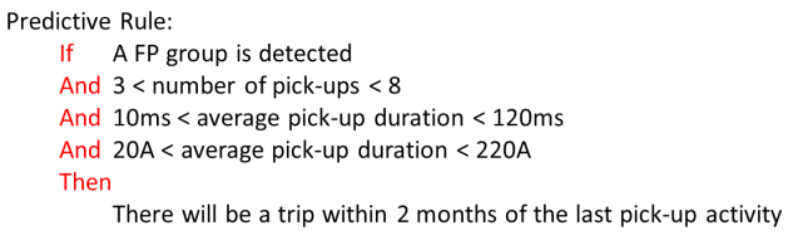

Fig. 6 Rule for predicting PMAR operation

With this generated rule, engineers can get an "early warning' report of potential PMAR operation. This allow experts to take evasive action and potentially prevent permanent outages resulted from SPFs.

\section{CASE STUDIES: IMPLEMENTATION OF THE VISUALISATION TOOL}

The diagnostic and prognostic functions integrated within the DSS were developed in a Java tool which included visualisation of results. The case studies demonstrate the functionalities outlined in the previous sections through application to a PMAR which experienced frequent supply interruptions.

After importing the particular PMAR log file, the KBS system will automatically identify PMAR device faults and generate a report through the DSS user interface. Fig. 7 shows the detection of the Microswitch fault explained with an unexpected accelerated contact time.

\begin{tabular}{|c|c|c|}
\hline PMAR device fault detection & 口回 & 23 \\
\hline PMAR Device Fault Detection Result & & \\
\hline $\begin{array}{l}\text { Mircoswitch Fault } \\
\text { - Due to an unexpected accelerated contact time }\end{array}$ & & \\
\hline
\end{tabular}

Fig. 7 Identification of Microswitch fault 
To detect the existence of SPFs, the KBS automatically classifies the stored fault information and provides the analysis through the user interface and visualisation tool. Fig. 8 illustrates the classification of PMAR operation based on the affected phases.

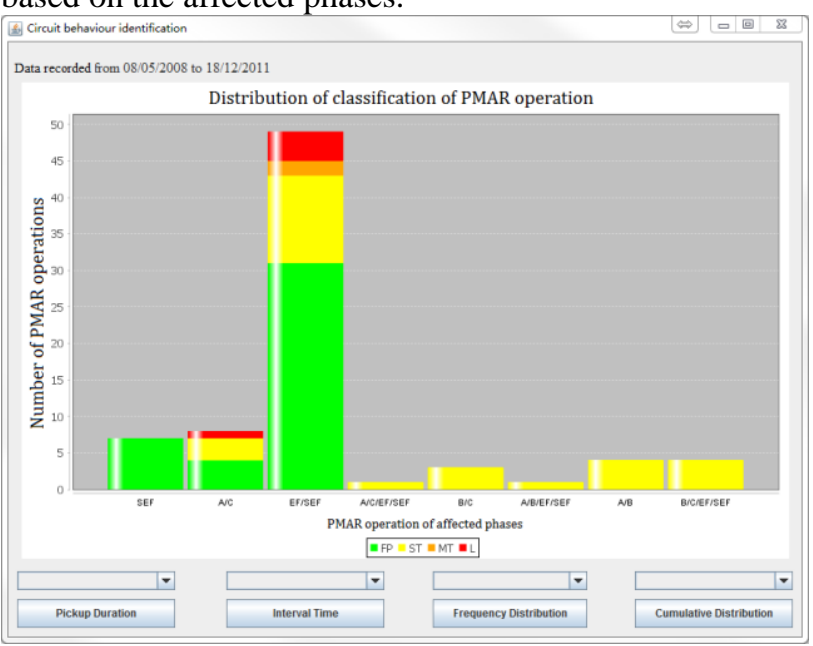

Fig. 8 Classification of PMAR operation

In Fig. 8, the different fault phases are listed with numbers and classifications of PMAR operation, marked by distinct colours. Obviously, Earth Fault and Sensitive Earth Fault (EF/SEF) is the main contributor of the number of operations in this particular PMAR. These indicate that frequent short-term fault activities could be caused by a SPF. For further analysis of the potential existence of SPFs, the key features (i.e. DT, TT, FD, CFD) will be selected and analysed. Fig. 9 shows the system's visualisations for the information of EF/SEF's FD and CFD.

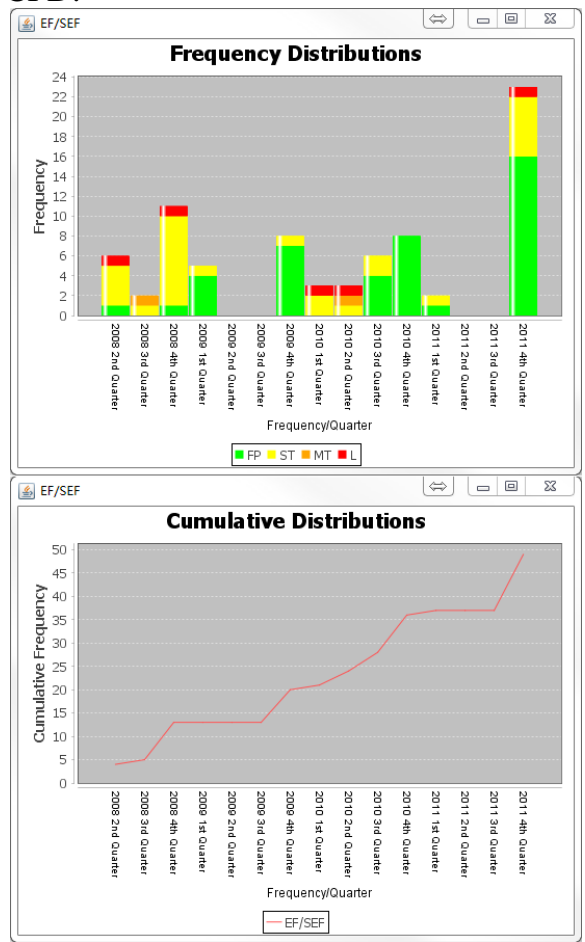

Fig. 9 The FD and CFD of EF/SEF
With the frequency distribution shown in Fig. 9, the PMAR always operates a single reclosure attempt to clear the transient fault. Meanwhile, the number of FP activities increase during the same period as well. Although the FP activity does not lead to a PMAR operation, the growing number indicates the increasing severity of fault conditions. The control engineers could conclude that the EF/SEF events could be arising from the existence of SPFs

Finally, the KBS application generates the fault prognosis report based on invoking the predictive rule. Fig. 10 shows the automatic prediction information delivered by the system.

Data recorded from 08/05/2008 to 14/12/2011
Prediction Result
Based on current data analysis, there could be a PMAR operation
in next 2 months from 14/12/2011 11:50:02:395

Fig. 10 Prediction for PMAR operation

\section{DISCUSSION AND CONCLUSION}

This paper offers a decision support system for automatic fault diagnosis and prognosis to mitigate customer supply interruptions and detect the underlying asset deterioration. The case studies demonstrate the processes and methodologies and how the automatic system assists the control engineers with fault data analysis. The automated analysis is supported by a flexible and effective visualisation tool integrated within the the system. This helps the engineers observe the processed fault information and assists their decision making.

The data analysis methodology can be applied to a wider set of DA devices, and the developed knowledge-base can be easily edited and extended as more data and knowledge/experience is generated.

\section{REFERENCES}

[1] A. J. McHarrie, 2012, "SP Energy Networks 20152023 Business Plan", SP Energy Networks, UK, 1819.

[2] J. A. Momoh, 2007, Electric Power Distribution, Automation, Protection, and Control, CRC Press, 18.

[3] NOJA POWER [Online], Available: http://www.nojapower.co.uk/product/recloser.html.

[4] A. MacGregor, 2012, "Application of Overhead Line Switchgear and Protection Systems", SP Energy Networks, UK, 7-12.

[5] SP Energy Networks, 2012, "Innovation Funding Incentive Annual Report", SP Energy Networks, UK, 14-17.

[6] X. Wang, S. M. Strachan, S. D. J. McArthur, and J. D. Kirkwood, 2015, "Automatic analysis of pole mounted auto-recloser data for fault diagnosis and prognosis", IEEE 18th ISAP conference, 1-6. 